inhaltlich-interpretativen Perspektive hinter diesen Strukturen steht, bleibt dabei häufig ein Forschungsdesiderat. Dabei ist es vor allem im Kontext der Relationen zwischen potentiell oppositionell zueinander stehenden Akteur_innen - wie in dieser Arbeit alternative im Gegensatz zu professionellen Nachrichtenmedien - hochrelevant, jene Beziehungen und entsprechend dazwischenliegende Narrative genauer $\mathrm{zu}$ betrachten, so beispielweise, wenn gesellschaftliche Problematiken wie das Echokammern-Phänomen abgeleitet werden (vgl. Kapitel II.3.3). Die Erforschung sozialer Netzwerkstrukturen hat zudem eine lange Tradition, vor allem im Bereich soziologischer Theorien. Aus theoretischer Sicht sind soziale Netzwerke nicht erst seit dem Aufkommen von Social-Media-Plattformen (als die sozialen Netzwerke moderner, digitaler Gesellschaften) ein zu untersuchendes Phänomen. Die Wurzeln liegen in soziologischen Klassikern, wie beispielsweise Max Weber (1985 [1922]), Georg Simmel (1968 [1908]), Norbert Elias (2006 [1970]) oder Pierre Bourdieu (2013 [1979]), deren theoretische Beiträge im Rahmen der relationalen Soziologie auf die Netzwerkanalyse angewendet werden können (Emirbayer, 1997; Häußling, 2010). Diese werden als besonders fruchtbar für die Analyse digitaler Plattformen und die dort auffindbaren Relationen zwischen Akteur_innen erachtet.

Vor diesem Hintergrund dient das vorliegende Kapitel einer theoretischen Auseinandersetzung mit dem Netzwerkbegriff aus einer relational-soziologischen Perspektive (Kapitel IV.1), die gleichzeitig eine Begründung des empirisch-methodischen Zugangs an das Forschungsobjekt dieser Arbeit darstellt. Im Anschluss folgt eine kritische Reflexion der aktuellen (digitalen) Netzwerkforschung (Kapitel IV.2).

\title{
IV.1 Relationale Soziologie und digitale Räume²
}

Der in Kapitel II.3.1 eingeführte Begriff der «Netzwerköffentlichkeit» impliziert die Annahme einer relationalen Betrachtung öffentlicher Kommunikation. Demnach können - wie auch Castells (2007, S. 238) im Rahmen seiner Ausführungen zur Netzwerkgesellschaft deutlich macht - Medien selbst als sozialer Raum betrachtet werden, in dem Macht ausgehandelt wird. Durch die Unterscheidung von Macht und Gegen-Macht («counter-power» (Castells, 2007, S. 239)) kann von einem relationalen Feld ausgegangen werden, in dem sich Akteur_innen, die diese oppositionelle Position einnehmen, gegen institutionalisierte Machtverhältnisse positionieren. Insofern entsteht ein Feld aus Akteur_innen mit unterschiedlicher Deutungsmacht: In seinen Extrempolen die hegemoniale Öffentlichkeit, z. B. in Form von Massenmedien und politischen Eliten, die aufgrund ihrer Reichweiten

2 Vgl. hierzu auch Schwaiger (2021 [im Erscheinen]). 
den Diskurs leitet, auf der anderen Seite Gegenöffentlichkeiten, die die Gegenposition einnehmen. Auszugehen ist allerdings von einem kontingenten Raum, wonach neben diesen konträren Polen weitere Akteur_innen Positionen im Feld innehaben - ähnlich der Longtail-Metapher (Eisenegger, 2021; Neuberger, 2009), die ein Spektrum an unterschiedlichen Kommunikator_innen gemessen an deren Reichweite annimmt (vgl. Kapitel II.3.2). Castells (2010 [1996]) spricht in seinen Werken schon früh von einer sogenannten «Netzwerkgesellschaft», die aus einer Perspektive der digitalen Transformation der Öffentlichkeit noch weiter an Bedeutung gewinnt. Durch die Etablierung digitaler Plattformen, die dezidiert der Vernetzung von Akteur_innen (Individuen, aber z. B. auch Organisationen) gewidmet sind, werden Netzwerkstrukturen in der Öffentlichkeit noch deutlicher. Dabei sind Plattformen einerseits als Raum dafür zu sehen, in dem zeit- und ortsunabhängig ohne Zugangsbarrieren öffentlichkeitswirksam agiert werden kann und weiter - die Sozialperspektive betreffend - Nutzer_innen individuelle Netzwerke mit auf der Plattform vertretenen Akteur_innen aufbauen können. $\mathrm{Zu}$ diesen Akteur_innen zählen nebst Individuen (Privatpersonen und Personen des öffentlichen Lebens) auch Organisationen und Institutionen; für den Kontext der vorliegenden Arbeit sind vor allem Social-Media-Auftritte von Nachrichtenmedien von Interesse.

Der Raum-Begriff selbst bedarf an dieser Stelle einer kurzen soziologischen Einordnung. Im Rahmen dieser Arbeit wird weniger an einen physischen, sondern vielmehr an einen abstrakt imaginierten Raum gedacht. So kritisiert beispielsweise Simmel (1905) im Rahmen seiner Kant-Vorlesungen Kants Vorstellung eines Raumes in Form eines Behälters (vgl. Zitat zu Beginn dieses Kapitels IV), wonach der «Raum selbst nichts Räumliches sei» (Simmel, 1905, S. 55) und vielmehr davon abhänge, welches menschliche Empfinden und Handlungen, also Inhalte, diesem gegeben würden (Löw, 2001, S. 58f.). Auch Löw (2001) grenzt sich in ihrer Theorie zur Raumsoziologie von einer absolutistischen Raumvorstellung, im Sinne eines geometrischen Raumes in Form eines Behälters, ab: «Die Entstehung eines Raums ist ein soziales Phänomen und damit nur aus den gesellschaftlichen Entwicklungen heraus, das heißt auch als prozeßhaftes Phänomen, zu begreifen.» (Löw, 2001, S. 263). Die Autorin ist folglich der Auffassung, dass Räume auf der Struktur menschlichen Handelns und sozialer (materieller) Güter beruhen und weniger als starre Gebilde $\mathrm{zu}$ interpretieren sind. Löw plädiert weiter für einen relativistischen, relationalen Raumbegriff, wobei diese Relationalität auf der Positionierung der Menschen im Raum gründet. Folglich stellen Individuen selbst Elemente dar, die zu Räumen verknüpft sind und deren Verknüpfung an menschliches Handeln gebunden ist (Löw, 2001, S. 224). Unter relationaler Anordnung versteht Löw, dass durch das Ensemble von Elementen bzw. deren Figuration (vgl. Norbert Elias' Figurationsbegriff, Kapitel IV.1.2) Räume erst konstruiert werden. «Syntheseleistung» meint in diesem Kontext, diese Ensembles sozialer 
Güter und Individuen als Einheit oder Element zu fassen. Das «Sich-Platzieren» oder «Spacing» bedeutet die Aushandlung der Position von Elementen innerhalb des Raumes. Die «Syntheseleistung» wie auch das «Spacing» bedingen sich gegenseitig, wonach die Verknüpfung und Positionierung der Elemente von der jeweiligen Handlungssituation abhängen. Die Konstitution des Raumes basiert zudem auch auf Vorstellungs-, Wahrnehmungs- und Erinnerungsprozessen (Löw, 2001, S. 225). Diese Aspekte treffen nicht nur auf den physischen Raum zu, sondern auch auf den virtuellen respektive digitalen Raum (Löw, 2001, S. 93). Gerade die Imagination des Raumes durch Individuen, $u$. a. basierend auf Erinnerungen und Vorstellungen, spielt hierbei eine wesentliche Rolle. Löws Raumtheorie ist im Kontext der digitalen öffentlichen Kommunikation vor allem deshalb interessant, da die Autorin den relationalen Aspekt der Raumkonstitution herausstreicht, der in ähnlicher Weise in der relationalen Soziologie im Kontext der Beschreibung von Netzwerken tragend ist. Insofern können Digitalplattformen als digitale Räume betrachtet werden, in denen bestimmte Akteur_innen Plätze einnehmen («Spacing»), im Sinne einer relationalen Anordnung dieser basierend auf sozialen Beziehungen, die in ihrer Summe den digitalen Raum erst definieren ( Syntheseleistung»).

Im Rahmen der aktuellen empirischen Erforschung von Digitalplattformen wird häufig der Eindruck erweckt, dass Analysen sozialer Netzwerke in diesem digitalen Kontext ihren Ursprung oder zumindest aktuelles Haupteinsatzgebiet fänden. Dabei handelt es sich bei der Untersuchung sozialer Netzwerke um eine grundlegende soziologische Frage, mit der sich die Soziologie seit jeher auseinandersetzt. Im Fokus stehen vorwiegend Relationen zwischen Individuen, die im Rahmen verschiedener soziologischer klassischer Schriften behandelt wurden, z. B. von Max Weber, Norbert Elias, Georg Simmel, Pierre Bourdieu u. a. So spricht beispielsweise Max Weber (1984 [1921], S. 1) von der Soziologie als einer «Wissenschaft, welche soziales Handeln deutend verstehen und dadurch in seinem Ablauf und seinen Wirkungen ursächlich erklären will». Soziales Handeln kann dementsprechend als relational betrachtet werden, da es immer auf das Verhalten anderer Akteur_innen bezogen ist und sich am Verhalten anderer orientiert (Weber, 1985 [1922], S. 11). Dabei unterscheidet Weber weiter zwischen zweckrationalem, wertrationalem, affektuellem und traditionalem Handeln. Zweckrationales Handeln orientiert sich an den Erwartungen des Verhaltens von anderen, um eigene, rationale Zwecke oder Erfolge zu erreichen. Wertrationales Handeln bezieht sich auf den eigenen Glauben, im Sinne von Ethik oder Religion. Affektuelles Handeln richtet sich nach der aktuellen Gefühlslage, den Emotionen. Traditionales Handeln steht im Zusammenhang mit Gewohnheiten (Weber, 1985 [1922], S. 12). Während zweck- und wertrationales Handeln in der sozialen Beziehung der «Vergesellschaftung» resultiert, im Sinne eines rationalen Interessenausgleiches oder Interessenverbindungen basierend auf Vereinbarung, definiert 
Weber «Vergemeinschaftung» als jene soziale Beziehungen, die auf affektuellem oder traditionellem Handeln, also einer subjektiv gefühlten Zusammengehörigkeit der Akteur_innen, beruhen (Weber, 1985 [1922], S. 21).

Auf Klassiker wie diese stützt sich auch die relationale Soziologie, die sich von der klassischen Netzwerkforschung abzugrenzen versucht. Die relationale Soziologie geht davon aus, dass Netzwerke nicht aus starren Strukturen bestehen, sondern aus Sinn und Narrativen in der Form von Sozialbeziehungen, als kleinste Einheit von Netzwerken (Mützel \& Fuhse, 2010, S. 8ff.; White, 1992). In der relationalen Betrachtung wird also weniger von Einzelakteur_innen und deren Bedürfnissen oder Kalkülen ausgegangen, sondern von relationalen Verbindungen, also Beziehungen zwischen Akteur_innen in Form von Netzwerkstrukturen und -dynamiken (Häußling, 2010). Entsprechend ist ein phänomenologischer Blick auf Netzwerke anzunehmen (Fuhse, 2008).

Obgleich die Wurzeln dieser Theorie- und Forschungsperspektive von klassisch soziologischen Schriften abgeleitet werden können, finden sie unter der Benennung als «relationale Soziologie» in den 1990er Jahren bei amerikanischen Soziologen wie Harrison White (1992) oder Mustafa Emirbayer (1997) ihren Ursprung. White (1992) beschreibt in «Identity and Control» seinen Ansatz der «phänomenologischen Netzwerktheorie», wonach strukturalistische Ansätze der Netzwerkforschung durch eine kulturelle Perspektive ergänzt werden. Soziale Beziehungen basieren demzufolge auf «kulturellen Annahmen und Interpretationen» (Mützel \& Fuhse, 2010, S. 13), also einer Bedeutungszuschreibung durch die Akteur_innen. Weitere Autoren argumentieren stärker aus einer Marktorientierung. So streicht beispielsweise Podolny (2001) die Bedeutung des individuellen Status in Netzwerkbeziehungen heraus, Granovetter (1973) wiederum verdeutlicht, dass auch schwache Verbindungen («weak ties») zwischen Akteur_innen individuelle Chancen beispielsweise am Arbeitsmarkt erhöhen. In ähnlicher Weise kann Emirbayers (1997) «Manifeso for a Relational Sociology» als Basiswerk der relationalen Soziologie betrachtet werden. Auch Emirbayer grenzt sich von einer strukturalistischen Sichtweise gegenüber Netzwerken ab - wie beispielsweise dem Rational-Choice-Ansatz (z. B. Burt [2000]) - und versteht Netzwerke respektive Relationen als etwas Dynamisches, Kontinuierliches und Prozesshaftes. In seinem «Manifest» nimmt der Autor Bezug auf klassisch soziologische Schriften, um seine Theorieperspektive zu verdeutlichen. Zentral ist hier Norbert Elias' (2006 [1970]) Figurationstheorie zu nennen, nach der Netzwerke als «Figurationen» betrachtet werden können, also Beziehungsgeflechte zwischen Individuen und Entitäten, in die Individuen hineinsozialisiert werden. Figurationen existieren nicht statisch, sondern sind als volatil wie auch einzigartig zu betrachten (Elias, 2006 [1970], S. 170-176; Schrape, 2013). Ein noch früherer Bezugspunkt zur relationalen Soziologie findet sich bei Georg Simmel (1968 [1908]). Dieser streicht in seinen Ausführungen zu den sozialen Kreisen die Individualität von Netzwer- 
ken heraus, wonach Individuen über einzigartige, spezifische Sets an sozialen Kreisen verfügen. Akteur_innen stehen demnach in einem wechselseitigen Verhältnis zueinander und sind in ihrem Handeln, der gegenseitigen Wahrnehmung und Erwartungshaltung voneinander abhängig (Häußling, 2010, S. 64f.). Als weitere klassische, wenn auch jüngere Quelle, soll in diesem Rahmen Bourdieus (2010 [1994]) Habitus- und Feldtheorie ergänzt werden. Unter «Feld» ist in diesem Kontext eine «Bündelung von Relationen zu verstehen» (Häußling, 2010, S. 68).

In den folgenden Subkapiteln werden die drei theoretischen Ansätze in chronologischer Reihenfolge (Simmels (1968 [1908]) «Soziale Kreise», Elias' (2006 [1970]) «Figurationstheorie» und Bourdieus (2013 [1979]) «Feldtheorie») genauer erörtert, mit dem Ziel, deren Bedeutung für die Netzwerkforschung - die wesentlich für diese Arbeit ist - zu verdeutlichen. Selbsterklärend handelt es sich dabei um keine abschliessende Erörterung von Theorieperspektiven, die prägend für die Ausgestaltung der relationalen Soziologie waren bzw. sind. Sie werden jedoch als besonders prägnant und anschaulich für die aktuelle Netzwerkforschung und insbesondere dieses vorliegende Projekt erachtet, da sie in sich zwar eigenständige Theorien bilden, in ihrer Summe aber verdeutlichen, inwiefern interpretative Zugänge für die Analyse von Beziehungsnetzwerken (unabhängig von deren Form, ob zwischen Individuen in Kopräsenz (physischer Raum) oder Akteur_innen auf digitalen Plattformen (digitaler Raum)) ergiebig sind, zumal die in den Theorien verwendeten Begrifflichkeiten für unterschiedliche empirische Wirklichkeitsausschnitte adaptierbar sind. Ziel der folgenden Kapitel ist es zudem, die wesentlichen Essenzen herauszufiltern, die für die Analyse der Netzwerköffentlichkeit als fruchtbar erachtet werden.

\section{IV.1.1 Georg Simmel: Soziale Kreise}

Georg Simmel (1968 [1908]), als ein Vertreter der «Klassiker» der Soziologie, beschäftigte sich im Rahmen seiner «Untersuchungen über die Formen der Vergesellschaftung» zu Beginn des 20. Jahrhunderts mit sozialen Beziehungen bzw. sozialen Kreisen. Er nimmt einen wesentlichen Stellenwert in der Netzwerkforschung ein, da in seinem Verständnis Individuen von Wechselwirkungen mit anderen Akteur_innen geprägt sind und diese Wirkungen selbst konstituieren, was dem Ansatz der relationalen Soziologie entspricht (Nollert, 2010, S. 157). Simmel geht davon aus, dass Individuen über spezifische Sets sozialer Kreise verfügen. Erscheint es noch rein zufällig, in welchen (familiären) Kreis Individuen per Geburt hineingeboren werden, werden die jeweiligen Kreise wesentlich heterogener, je nachdem, mit welchen Persönlichkeiten ausserhalb dieses Kreises Beziehungen geknüpft werden, im Sinne einer Gemeinschaftsbildung (vgl. hierzu auch Webers Gemeinschaftsbegriff in diesem Hauptkapitel). Nach Simmel (1968 [1908], S. 305) steht folglich ein_e jede_r mit seinem oder ihrem Umfeld in einem «assoziativen 
Zusammenhang». Dabei beginnt die Umgebung von Individuen zunächst mit dem Zusammensein von Verbindungen mit Individuen, die durch Geburt (im Sinne eines Zufalls) zu ihnen gestellt sind - also dem primären sozialen Kreis -, der im Verlauf des Lebens (und zunehmender sozialer Ausdifferenzierung) durch heterogene Kreise im Sinne von Relationen mit unterschiedlichen Akteur_innen ergänzt wird, die ausserhalb des primären Assoziationskreises liegen: «der ursprüngliche Zusammenhang der Familiengruppe wird dadurch modifiziert, daß die Individualität des Einzelnen diesen in anderweitige Kreise einreiht» (Simmel, 1968 [1908], S. 309f.). Jene Beziehungen basieren Simmel zufolge auf ähnlichen Anlagen, Neigungen oder Tätigkeiten, wonach vor allem inhaltliche oder auch sinnliche Beziehungen an Bedeutung gewinnen. Zusammengehörigkeit kann folglich auf Zweck und Rationalisierung beruhen, also innerlich-sachlichen Gesichtspunkten, oder auf individuellem Interesse, denkbar beispielsweise in beruflichen Kreisen und einer Gleichheit in Bezug auf die Beschäftigung (Simmel, 1968 [1908], S. 311). Diese auf (z. B. kulturellen, wirtschaftlichen oder politischen) Interessen beruhenden Kreise befördern «Homophilie», worunter eine Bevorzugung von gleichgesinnten Menschen zu verstehen ist (McPherson, Smith-Lovin \& Cook, 2001; Nollert, 2010, S. 158). Neben wirtschaftlichen, zweckmässigen Kreisen (oder bei Simmel auch: kriegerischen oder politischen) und familiären Kreisen, erwähnt Simmel auch gefühlsmässige, also religiöse Verbindungen (Simmel, 1968 [1908], S. 311). In modernen Gesellschaften können soziale Kreise häufig frei gewählt werden, auch ist die Anzahl individueller Kreise in der Moderne deutlich höher: «Die Zahl der verschiedenen Kreise nun, in denen der Einzelne steht, ist einer der Gradmesser der Kultur» (Simmel, 1968 [1908], S. 311). So können auch einzelne Kreise, wie beispielsweise der berufliche Kreis, noch weiter ausdifferenziert oder ergänzt werden, z. B. durch die gefühlte Zugehörigkeit zu einem sozialen Stand. Damit können sich auch Individuen, die demselben sozialen Kreis angehören, über einzelne entferntere Kreise voneinander abgrenzen. Die Summe aller Kreise kann als eine Art Koordinatensystem gedacht werden, mit dem sich die Position von Individuen durch jedes Hinzukommen weiterer Kreise noch eindeutiger bestimmen lässt. Gleichbedeutend ist es denkbar unwahrscheinlich, dass andere Personen über die exakt selbe Gruppenkonstellation verfügen, woraus sich die Individualität von Einzelpersonen ergibt - nämlich der Kreuzungspunkt aller sozialer Kreise. Das Aufeinanderprallen diverser Gruppeninteressen erachtet Simmel (1968 [1908], S. 312f.) als konstitutiv für die Einheit, also die eigene Persönlichkeit. Für die Individualisierung ist es zudem essentiell, dass Individuen innerhalb ihrer Kreise unterschiedliche relationale Stellungen einnehmen können, d. h. in bestimmten Kreisen zentralere Positionen und in anderen peripherere (Simmel, 1968 [1908], S. 319). Neben diesem Individualismus streicht Simmel (1968 [1908], S. 325f.) auch einen gleichzeitigen Kollektivismus heraus, im Sinne eines dahingehenden Dualismus: 
Denn einerseits findet der Einzelne für jede seiner Neigungen und Bestrebungen eine Gemeinschaft vor, die ihm die Befriedigung derselben erleichtert, seinen Tätigkeiten je eine als zweckmäßig erprobte Form und alle Vorteile der Cruppenangehörigkeit darbietet; andrerseits wird das Spezifische der Individualität durch die Kombination der Kreise gewahrt, die in jedem Fall eine andre sein kann. So kann man sagen: aus Individuen entsteht die Cesellschaft, aus Cesellschaften entsteht das Individuum.

Demzufolge sind soziale Kreise einerseits dadurch geprägt, dass sie (meist) frei und individuell gewählt werden können. Andererseits ergibt sich gerade die Individualität aus der Überschneidung sozialer Kreise. Das Agieren in unterschiedlichen Kreisen impliziert gleichzeitig Intra- und Interrollenkonflikte, da in unterschiedlichen Kreisen verschiedene Erwartungen und Ansprüche an Individuen gerichtet werden (Nollert, 2010, S. 158). Dabei steigt die Komplexität dieser Erwartungen durch die Anzahl aber auch die Grösse der Kreise, oder umgekehrt: Je kleiner der soziale Kreis (wie beispielsweise der primäre Kreis der Familie), umso konkreter die Rollenerwartungen (Abels \& König, 2016, S. 11). Diese wechselseitigen Erwartungen sind essentiell für die Vergemeinschaftung, gleichzeitig können diese als konstitutiv für relationale Verbindungen betrachtet werden. Die Schnittstelle sozialer Kreise gibt so die Position im «Netzwerk» von Individuen an.

\section{IV.1.2 Norbert Elias: Figurationen}

Mit wechselseitigen, relationalen Bezugnahmen zwischen Individuen beschäftigt sich auch Norbert Elias (2006 [1970]), wenn auch mit einer anderen Begriffsarchitektur. Elias stellt sich in seinen Ausführungen zur Figuration die Frage, inwiefern Individuen wechselseitig voneinander abhängig sind - zumal er davon ausgeht, dass Individuen nicht ohne ihre Relationen $\mathrm{zu}$ anderen Menschen betrachtet werden können (Treibel, 2008, S. 69). Im Kontext der Analyse von Figurationen nach Elias treten das Individuum bzw. die Verflechtungen von Individuen in den Fokus der Betrachtung. Dabei nimmt Elias (2006 [1970], S. 171) weniger eine dichotome Betrachtung von Individuum einerseits und Gesellschaft andererseits an, sondern sieht diese vielmehr als «unabtrennbare Ebenen des menschlichen Universums». Der Begriff «Figuration» beschreibt demnach interdependente Handlungen zwischen Individuen, oder «Beziehungsgeflechte von Menschen, die mit der wachsenden gegenseitigen Abhängigkeit der Menschen untereinander immer komplexer werden» (Treibel, 2008, S. 70). Da Figurationen situationsbedingt wechseln können, spricht Elias (2006 [1970], S. 174) auch von «Figurationsprozessen», die beispielsweise auch fluktuierende Machtbalancen zwischen Akteur_innen implizieren würden. Elias nimmt weiter Abstand von egozentrierten Gesellschaftsbildern, in denen das «Ich» umgeben von der Gesellschaft respekti- 
ve sozialen Gebilden sei (z. B. Familie, Staat). Vielmehr seien Individuen untereinander durch unterschiedliche Weise miteinander verbunden, basierend auf Machtbalancen und verschiedenen affektiven Bindungen respektive «Valenzen» (Elias, 2006 [1970], S. 14f.).

Elias stellt im Rahmen seiner Ausführungen des Figurationsbegriffes folgende zentrale Frage: «Was ist es, das Menschen aufeinander angewiesen, voneinander abhängig macht?» (Elias, 2006 [1970], S. 177). In diesem Zusammenhang identifiziert er unterschiedliche Arten von Bindungen zwischen Individuen; darunter emotionale, affektive Bindungen z. B. in intimen Beziehungen oder aber staatliche und berufliche Bindungen. Affektive Bindungen basieren ihm zufolge auf tief in Individuen verwurzelten, emotionalen Bedürfnissen nach Angehörigkeit in der Gesellschaft, wobei sich das stärkste Bedürfnis diesbezüglich in der Sexualität, als biologischem Antrieb, widerspiegele. Elias (2006 [1970], S. 181) sieht diese persönlichen Interdependenzen als wesentliches «Bindemittel der Gesellschaft» und distanziert sich beispielsweise von Ansätzen, die vor allem wirtschaftliche Bindungen als notwendige Folge der zunehmenden Spezialisierung der Arbeit und damit einhergehenden Angewiesenheit der Menschen untereinander herausstreichen, wie beispielsweise bei Emile Durkheim. Emotionale Bindungen nach Elias beschränken sich weiter nicht nur auf Beziehungen zwischen Menschen, sondern können auch, wenn von grösseren gesellschaftlichen Einheiten gesprochen wird, auf Symbole des Staates wie Wappen oder Fahnen ausgedehnt werden. Eine emotionale Bindung zwischen Individuen kann sich dementsprechend nicht nur in Form von Face-to-face-Beziehungen ergeben, sondern auch vermittelt über Symbole, die sie aneinanderbinden. Dadurch entsteht ein «Ich-und-Wir» -Bewusstsein, das sich über Millionen umfassende Einheiten ausdehnen kann (Elias, 2006 [1970], S. 182). Staatliche und berufliche Interdependenzen beziehen sich wiederum auf die gesellschaftliche Bindungskraft von Staaten respektive Stämmen, da diese als «Objekte gemeinsamer Identifizierungen» betrachtet werden können (Elias, 2006 [1970], S. 183). Dabei schaffe der Staat - so Elias (2006 [1970]) - Bindungen dadurch, dass vor physischer Gewalt einerseits durch Kontrolle unter den Gesellschaftsmitgliedern geschützt werde, andererseits werde auf den Einsatz von Gewalt gegenüber Nichtangehörigen vorbereitet, im Sinne von Zusammenschlüssen zwischen Individuen, um ihre eigene Interessen respektive ihr Leben vor dem Angriff anderer Gruppen zu schützen. Eng mit staatlichen Interdependenzen verbunden sind nach Elias wirtschaftliche bzw. berufliche Verflechtungen, da sich staatliche Einrichtungen im Gleichgang mit wirtschaftlichen Einrichtungen ausdifferenzieren, beispielsweise um Machtbalancen in der Wirtschaft (z. B. zwischen Unternehmer- und Arbeitergruppen) zu gewährleisten.

Diese Figurationen sind nicht statisch und können sich gleichbedeutend durch eine relative, aber nicht absolute Autonomie von Individuen gegenüber diesen Verflechtungszusammenhängen wandeln. Elias (2006 [1970], S. 267) geht 
davon aus, dass Figurationen eine gewisse Macht über Individuen haben und sie «in ihrer Freiheit beschneiden». Diese Macht liegt folglich nicht ausserhalb der Individuen, sondern basiert auf den jeweiligen Interdependenzen. Elias lehnt Ansätze $a b$, die annehmen, dass bestimmte Individuen über Macht als eine Art Eigenschaft oder Ressource verfügen - erst deren Relation zu anderen Individuen macht sie mächtig und auch diese Macht ist nicht statisch (Treibel, 2008, S. 75). Vielmehr geht Elias (2006 [1970]) von dynamischen Machtverhältnissen, d. h. einem Wechselspiel zwischen Macht und Gegenmacht, anstelle von einseitigen Abhängigkeiten aus. In diesem Kontext unterscheidet er auch zwischen Etablierten und Aussenseiter_innen (Elias \& Scotson, 2002[1965]). Die gleichnamige Studie setzt sich mit den Figurationen von alten (ansässigen) und neuen Familien in Winston Parva gegen Ende der 1950er Jahre auseinander. Die Autoren beschreiben hier starke Verflechtungen respektive einen Gruppenzusammenhalt innerhalb der Gruppen, während Neuankömmlinge ausgeschlossen worden seien und Macht ein Monopol erhalten habe. Im Laufe der Zeit hätten die Neuankömmlinge die ihnen herangetragenen negativen Attribute verinnerlicht und seien auch in ihrer Selbstwahrnehmung zu den Aussenseiter_innen geworden. Diese gegenseitigen Zuschreibungen hätten schliesslich das Machtgefälle zwischen den Gruppen forciert, während das «Wir-Gefühl» stärker geworden sei. Elias und Scotson bezogen sich im Rahmen der Studie zwar primär auf Familienverhältnisse, das Etablierten-Aussenseiter_innen-Konzept kann allerdings auch beispielsweise auf Staaten (z. B. Migration) und weitere Systeme adaptiert werden (vgl. hierzu auch Treibel, 2008, S. 80-84). Für die vorliegende Studie erscheint das Konzept zudem wesentlich, um Gruppendynamiken und -prozesse hinsichtlich Ingroupund Outgroup-Prozesse - wie es bei Gegenöffentlichkeiten der Fall sein kann - zu beschreiben. Elias' (2006 [1970]) Figurationstheorie wurde entsprechend auch als Basis für die aktuelle Netzwerkforschung adaptiert; so kann der Netzwerkbegriff für die «Beschreibung jener dynamischen, veränderlichen und (hyper-)komplexen Figurationen, die sich, von Akteuren ausgehend und Akteure konstellierend, im Zuge der Mediatisierung bzw. Internetisierung der Gesellschaft entwickelt haben und entwickeln» gebraucht werden (Willems, 2010, S. 260f.).

\section{IV.1.3 Pierre Bourdieu: Feldtheorie}

Auch Bourdieus (2010 [1994], 2013 [1979]) Habitus- und Feldtheorie wird häufig in der Netzwerkforschung als Metatheorie genutzt (z. B. Bernhard, 2010; Fuhse, 2009; Hennig \& Kohl, 2012). Die Theorie überzeugt vor allem dadurch, dass sie die Mikro- mit der Makroebene verknüpft. So heben auch Hennig und Kohl (2012) deren relational soziologische Perspektive hervor, wonach Netzwerke zwar aus einer Makroperspektive soziale Strukturen deutlich machen, diese allerdings auch individuelle Interaktionsbeziehungen und eine damit einhergehende Mikropers- 
pektive sozialen Handelns miteinschliessen. Bourdieus Theorie eigne sich insofern, so die Autor_innen weiter, als der individuelle Habitus in Kombination mit der strukturellen Betrachtung eines Feldes beide Ebenen miteinander verknüpfe. So schreibt Bourdieu (1998 [1985], S. 15) selbst: «Das Reale ist relational». Insofern widersetzt sich Bourdieu mit seinem «primacy of relations» der gängigen theoretischen wie auch methodischen Tendenz, eine klare Trennlinie zwischen dem Kollektiven und dem Individuellen oder der Struktur und dem Handeln zu setzen, und reiht sich dabei in die relationalen Ansätze, z. B. von Norbert Elias, ein (Bourdieu \& Wacquant, 1992, S. 15) ${ }^{3}$. Sowohl das Feld als auch der Habitus werden bei Bourdieu als relationale Grössen betrachtet:

A field consists of a set of objective, historical relations between positions anchored in certain forms of power (or capital), while habitus consists of a set of historical relations «deposited» within individual bodies in the form of mental and corporeal schemata of perception, appreciation, and action. (Bourdieu \& Wacquant, 1992, S. 16)

Während also das Feld auf Macht- respektive Kapitalstrukturen beruht, die u. a. auf historischen Relationen basieren, bezieht sich der Habitus auf die individuellen Wahrnehmungs- und Handlungsmuster, die wiederum auf im Körper manifestierten Relationen fussen. Das Feld ist gleichzeitig als Aushandlungsort für Konflikte und Kompetition anzusehen (Bourdieu \& Wacquant, 1992); zentrale Positionen im Feld können als Ausdruck von Macht respektive Deutungsmacht interpretiert werden. Ein Feld bezieht sich dabei auf einen Ausschnitt der sozialen Realität, ähnlich einer systemtheoretischen Perspektive, die zwischen unterschiedlichen sozialen Systemen unterscheidet. So spricht auch Bourdieu von verschiedenen gesellschaftlichen Feldern, z. B. der Wissenschaft, Kunst oder Politik. Im Gegensatz zu systemtheoretischen Ansätzen, wie beispielsweise jenem nach Luhmann, fokussiert Bourdieu allerdings auf die Konstellation von Individuen (Fuhse, 2009). Das Feld repräsentiert eine Art Spielfeld der Machtaushandlung im sozialen Raum, innerhalb dessen die jeweilige Position der Individuen anhand von zwei respektive auch drei Unterscheidungsdimensionen offensichtlich wird: anhand des Kapitalvolumens, der Kapitalstruktur und allenfalls der zeitlichen Entwicklung (abgeleitet durch die soziale Laufbahn) dieser beiden Dimensionen

3 Bourdieu gehe demnach davon aus, dass sich diese duale oder dichotome Herangehensweise schon in unserer Sprache manifestiere, die besser Zustände denn Prozesse oder eben auch besser Dinge als Relationen ausdrücken könne. Von dieser dualen Wahrnehmung sozialer Realität müsse sich die Soziologie entsprechend distanzieren (Bourdieu \& Wacquant, 1992, S. 15). Ebenso verhält es sich im Übrigen meiner Ansicht nach mit dem Öffentlichkeits- und Gegenöffentlichkeitsbegriff oder: dem Mainstream und der Alternative, die nicht getrennt voneinander betrachtet werden können, da sie in einem relationalen Verhältnis zueinanderstehen. 
(Bourdieu, 1998 [1985], S. 18ff.; 2013 [1979], S. 195f.). Das Gesamtvolumen des Kapitals summiert sich aus den drei von Bourdieu entwickelten Kapitalsorten, dem ökonomischen, kulturellen, sozialen und symbolischen Kapital. Während sich das ökonomische Kapital vor allem auf materielle Ressourcen (z. B. Vermögen, Einkommen) bezieht, repräsentiert das kulturelle Kapital einerseits den Besitz kultureller Güter (z. B. Kunstwerke; auch objektiviertes Kulturkapital), kulturelles Wissen und Fähigkeiten (Bildung; auch inkorporiertes Kulturkapital) wie auch tatsächliche Bildungsabschlüsse (in Form von Bildungstiteln, Zertifikaten; auch institutionalisiertes Kulturkapital). Das Sozialkapital bezieht sich weiter auf persönliche Beziehungsnetzwerke in Form von Sozialbeziehungen wie der Familie, aber auch durch die Teilhabe an bestimmten gesellschaftlichen Gruppen (z. B. Organisationen). Das symbolische Kapital nimmt insofern eine besondere Stellung im Vergleich zu den restlichen Kapitalsorten ein, als es eine übergeordnete Rolle spielt und aus dem ökonomischen, kulturellen und sozialen Kapital emergiert. So kann durch den Einsatz der drei Kapitalsorten Prestige (oder auch Reputation) resultieren, das das symbolische Kapital definiert (Bourdieu, 2013 [1979]). Das symbolische Kapital ist demnach «eine beliebige Eigenschaft (eine beliebige Kapitalsorte, physisches, ökonomisches, kulturelles, soziales Kapital), wenn sie von sozialen Akteuren wahrgenommen wird, deren Wahrnehmungskategorien so beschaffen sind, daß sie zu erkennen (wahrzunehmen) und anzuerkennen, ihr Wert beizulegen, imstande sind.» (Bourdieu, 1998 [1985], S. 108). Symbolische Macht entsteht folglich durch die Wahrnehmung der jeweiligen Kapitalsorten in der Gesellschaft.

Jede Positionsklasse im sozialen Raum verfügt Bourdieu (1998 [1985], S. 20-23) zufolge zudem über einen bestimmten Habitus, oder auch Geschmack. Die jeweilige Positionierung wird über diesen Habitus vermittelt, der gleichzeitig auch Akteur_innen einer Klasse miteinander verbindet. Unter Habitus versteht Bourdieu (1998 [1985]) unterschiedliche Praktiken, Wahrnehmungen oder Geschmäcker als Distinktionsmerkmale ${ }^{4}$. Der individuelle Habitus ist einerseits gruppenintegrierend, andererseits wird dadurch der Unterschied gegenüber anderen Gruppen (oder Klassen) deutlich gemacht und schliesslich von anderen wahrgenommen.

Bourdieus (1998 [1985], 2013 [1979]) Habitus- und Feldtheorie beschäftigt sich zusammenfassend mit der «Bündelung von Relationen». Die auf dem Feld bestehenden Positionen bilden ein Ensemble aus Relationen, die auf Macht bzw. Kapital beruhen. Bourdieu (2010 [1994]) zufolge besteht die soziale Wirklichkeit deshalb ausschliesslich aus sozialen Relationen (vgl. hierzu auch Häußling, 2010).

4 »[...] was der Arbeiter ißt und vor allem, wie er ißt, welchen Sport er treibt und wie er ihn treibt, welche politischen Meinungen er hat und wie er sie zum Ausdruck bringt, unterscheidet sich systematisch von den entsprechenden Konsum- und Verhaltensgewohnheiten der Unternehmer in der Industrie.» (Bourdieu, 1998[1985], S. 21) 
Infolgedessen sind Individuen im öffentlichen wie auch im privaten Leben als Repräsentant_innen objektiver Positionen im sozialen Raum zu sehen (Blatter, Janning \& Wagemann, 2007). Für die Netzwerkforschung ist vor allem Bourdieus Betrachtung sowohl von Mikro- (Habitus) als auch Makrostrukturen (Feld) relevant. Der Strukturalismus der Netzwerkforschung wird somit durch die Perspektive sozialer Praktiken von Akteur_innen ergänzt. Die Struktur, in Form von unterschiedlichen Kapitalverteilungen, bestimmt massgeblich mit, wie sich subjektive Beziehungen konstruieren. Wesentlich für die Strukturierung des Feldes sind zudem Machtbeziehungen, die sich aus den unterschiedlichen Kapitalsorten ergeben und Gruppenprozesse auslösen können, im Sinne von Sieger_innen und Verlierer_innen innerhalb eines Feldes (Hennig \& Kohl, 2012, S. 21). Für die Netzwerkforschung ist weiter die Frage wesentlich, ob inkorporierte Denk- und Handlungsmuster (der Habitus) dazu führen, dass Akteur_innen vorwiegend innerhalb ihrer Klasse respektive mit ähnlichen sozialen Positionen interagieren. Der Habitus würde dann als Bindeglied innerhalb von Gruppen fungieren (Hennig \& Kohl, 2012).

\section{IV.2 Relationen auf digitalen Plattformen}

Wie im letzten Kapitel beschrieben, können klassisch soziologische Beiträge herangezogen werden, um Netzwerkdynamiken zu beschreiben. Dies ist für die vorliegende Arbeit von hoher Relevanz, zumal die digitale Öffentlichkeit Untersuchungsgegenstand ist, um das Phänomen von Gegenöffentlichkeiten zu analysieren. Zugrundeliegend ist die Annahme, dass digitale Öffentlichkeiten durch einen Netzwerkcharakter geprägt sind. Dies ist insbesondere im Zusammenhang mit der Untersuchung von Digitalplattformen, die einen wesentlichen Stellenwert in der Transformation der Öffentlichkeit einnehmen, relevant. Die folgenden Kapitel sollen aufzeigen, inwiefern digitale Öffentlichkeiten aktuell erforscht werden und wie diese Ansätze kritisch zu reflektieren sind (Kapitel IV.2.1). Abschliessend erfolgt eine Einordnung relational soziologischer Ansätze für die Erforschung von Digitalplattformen (Kapitel IV.2.2).

\section{IV.2.1 Aktuelle Ansätze zur Untersuchung digitaler Netzwerke ${ }^{5}$}

Obwohl die Erforschung von Netzwerken auf eine lange Tradition zurückblickt, hat sie in der empirischen Sozialforschung durch die Etablierung von Social-Media-Plattformen eine neue Rahmung erfahren. Populär wurde in diesem Zusammenhang vor allem die Analyse der Digitalplattform Twitter, die eine Datenerhe-

5 Dieses Kapitel bezieht sich vorwiegend auf Schwaiger (2021 [im Erscheinen]). 